\title{
Expression of TWEAK on the Ectopic and Eutopic Endometrium from Women with Endometriosis
}

\author{
Qian PENG, Jin ZHU \\ Obstetrics and Gynecology Hospital, Fudan University, Shanghai 200011, China
}

Objective To investigate the relationship between Tumor necrosis factor (TNF)-like weak inducer of apoptosis(TWEAK) and endometriosis.

Methods TWEAK $m R N A$ and protein concentrations in paired samples of eutopic endometrial tissue from women with and without endometriosis, and also of ectopic endometrial tissue from women with endometriosis were measured by Real-time PCR, immunohistochemistry and Western blotting, respectively.

Results Compared with control endometrium from women without endometriosis and eutopic endometrium from women with endometriosis, TWEAK expressions were reduced on the ectopic endometrium $(P<0.05)$. Moreover, the expressions of TWEAK $m R N A$ on eutopic endometrium and control endometrium in proliferative phase were much lower than those in secretory phase. TWEAK protein was expressed in the cytoplasm of glandular cells and stromal cells of endometrium.

Conclusion TWEAK is expressed on the endometrium of reproductive women, and its concentration rises in secretory phase, compared with that in proliferative phase. Patients with endometriosis had a lower expression of TWEAK on ectopic endometrium, which may lead to a decreased level of apoptosis on endometrial cells. Consequently, endometrial cells were able to survive outside uterus helping the development of endometriosis.

Key words: endometriosis (EMs); endometrium; TWEAK

Tumor necrosis factor (TNF)-like weak inducer of apoptosis (TWEAK) is the member of TNF cytokine superfamily. It could bind to different receptors specifically just as what TNF and FasL do to TNFR1 and Fas, and it has a great effect on the death signal transmitting and apoptosis induction, especially on causing tumor cells' death, inhibiting tumor growth and maintaining the homeostasis of immune system. The receptors of TWEAK include Fn-14, 
TRAMP (also known as DR3) and others. The interaction between them displays various biological functions ${ }^{[1]}$. Nakayama et al. have observed that TWEAK induced cell death in Fn-14 transfectants ${ }^{[2]}$. As far as we are concerned, endometrial cells of endometriosis are similar to tumor cells to a certain extent. For instance, endometrial cells can transfer far away and they also need formation of new vessels, both of which are necessary characteristics for tumor progression. Then, could TWEAK induce the apoptosis of immune cells and involve in the development of endometriosis through binding to its receptors, like FasL? We investigate the expression of TWEAK on the ectopic and eutopic endometrium from women with endometriosis, in order to evaluate the relationship between TWEAK and endometriosis.

\section{Materials \& Methods}

\section{Patients and samples}

All samples were obtained after informed consent of all patients, and the protocol was approved by the Ethical Committee of Obstetric and Gynecology Hospital. Eutopic endometrium ( $n=6$, proliferative phase; $n=6$, secretory phase) and ectopic endometrium $(n=29)$ were collected from patients undergoing laparoscopic surgery for endometriosis and hysterectomy for adenomyosis (Obstetric and Gynecology Hospital, Fudan University) at different phases of the menstrual cycles. These patients were selected from November 2006 to July 2007, who aged from 20 to 50 years ( $36 \pm 7$ years), and they were having a regular menstrual cycle of $26^{-}$ $35(28 \pm 3) \mathrm{d}$ without taking exogenous hormones within 3 months before the procedure. The samples of control group ( $n=8$, proliferative phase; $n=6$, secretory phase) were obtained from women without endometriosis who had hysterectomy for uterine myoma and pathological changes of cervix. They aged from 40 to 50 years ( $44 \pm 7$ years), with a regular menstrual cycle of 28-36 (29 \pm 3$) \mathrm{d}$. Exclusion criteria in both groups were absence of cancer and any other acute or chronic disease for the last 3 months. A portion of each sample was processed for histologic confirmation, and the remainders were immediately frozen in liquid nitrogen, some of which were fixed immediately in formalin (10\%) and then processed as paraffin blocks as well.

\section{RNA extraction}

Total RNA from 50-100 mg of endometrial tissue samples was extracted by Trizol reagent (Molecular Research Center, USA) following manufacturer's instructions. The RNA purity was verified by $\mathrm{A}_{260} / \mathrm{A}_{280}$ using ultraviolet spectrophotometer, which was supposed to result in $1.8-2.0$.

\section{RT-PCR}

Totally $2.5 \mu \mathrm{g}$ of RNA was reverse transcribed using RevertAid ${ }^{\mathrm{TM}}$ first strand cDNA synthesis kit (Fermentas, USA). The polymerase chain reaction (PCR) analysis was subject to validation and optimization steps to ensure that all reactions were linear relative to the amount of input cDNA and cycles of product amplification. All PCR reactions included 
12.5 $\mu 1$ PerfectShot ${ }^{\mathrm{TM}}$ Ex Taq (TaKaRa, Japan), $0.5 \mu 1$ TWEAK (Shanghai Sangon Biological Engineering Technology \& Services Corporation, China) and 0.5 $\mu$ GAPDH (Shanghai Sangon Biological Engineering Technology \& Services Corporation, China), $2 \mu$ cDNA. Final PCR conditions were defined as follows: denaturation program at $94^{\circ} \mathrm{C}$ for $10 \mathrm{~min}, 35$ cycles of denaturation at $94^{\circ} \mathrm{C}$ for $30 \mathrm{~s}$, annealing for $30 \mathrm{~s}$ at $58^{\circ} \mathrm{C}$ and elongation at $72^{\circ} \mathrm{C}$ for $45 \mathrm{~s}$, then elongation program at $72^{\circ} \mathrm{C}$ for $10 \mathrm{~min}$ for TWEAK and GAPDH. As for Fn-14, other programs are the same except for the annealing temperature is $54^{\circ} \mathrm{C}$. PCR products were tested by $2 \%$ agarose gel electrophoresis and photographed using an ultraviolet (UV) light source.

\section{Real-time PCR}

Semi-quantification of the mRNA coding for TWEAK (TaKaRa, Japan) and $\beta$-actin (TaKaRa, Japan) were performed using ABI PRISM ${ }^{\circledR} 7000$ Real-time PCR System (Applied Biosystems, USA). The reactions were performed in a volume of $25 \mu 1$ of a mixture containing SYBR ${ }^{\circledR}$ Premix Ex Taq ${ }^{\mathrm{TM}}(2 \times) 12.5 \mu$, ROX reference dye II $(50 \times) 0.5 \mu 1$ and cDNA $2 \mu$ l. The following Real-time PCR protocol was used: denaturation program at $95^{\circ} \mathrm{C}$ for $10 \mathrm{~s}, 40$ cycles of denaturation at $95^{\circ} \mathrm{C}$ for $5 \mathrm{~s}$, annealing for $31 \mathrm{~s}$ at $60^{\circ} \mathrm{C}$ for TWEAK and $\beta$-actin. To avoid detection of non-specific PCR products, the purity of each amplified product was confirmed by melting curve analysis according to manufacturer's manual. To prepare the standard curves, one of the sample cDNA was diluted by $1: 10$, $1: 100,1: 1000,1: 10000$ and $1: 100000$ for the substitute of the standard sample. For the PCR run, a standard curve was established using these serial dilutions of each corresponding external standard. For each example, the derived concentration was calculated from the standard curve by the instrument software. Results were presented as the ratio of the amounts of TWEAK amplification product over $\beta$-actin amplification product according to the cycles of threshold for each sample. These ratios were called TWEAK relative transcript or mRNA levels. Each value was calculated as an average of three independent PCRs. Experiments were conducted under identical conditions, and results were presented as $\bar{x} \pm s$.

Table 1 Primer sequence of PCR

\begin{tabular}{|c|c|c|c|}
\hline \multirow{2}{*}{$\begin{array}{l}\text { Gene } \\
\text { PCR }\end{array}$} & \multicolumn{2}{|r|}{ Primer sequence } & cDNA length (bp) \\
\hline & & & \\
\hline \multirow[t]{2}{*}{ TWEAK } & Forward & 5'-CCCTGCGCTGCCTGGAGGAA-3' & 200 \\
\hline & Reverse & 5'-AGACCAGGGCCCCTCAGTGA-3' & \\
\hline \multirow[t]{2}{*}{ Fn-14 } & Forward & 5'-TGGGGCCTAGTGTCAAGTCT-3' & 260 \\
\hline & Reverse & 5'-CCAAGCTCCTCCAACCACAA-3' & \\
\hline \multirow[t]{2}{*}{ GAPDH } & Forward & 5'-ACCACAGTCCATGCCATCAC-3' & 452 \\
\hline & Reverse & 5'-TCCACCACCCTGTTGCTGTA-3' & \\
\hline \multicolumn{4}{|c|}{ Real-time PCR } \\
\hline \multirow[t]{2}{*}{ TWEAK } & Forward & 5'-GAAGCCAGAATCAACAGCTCCAG-3' & / \\
\hline & Reverse & 5'-CCAGCAAGTCCAGCTTCAGGTAG-3' & \\
\hline \multirow[t]{2}{*}{$\beta$-actin } & Forward & 5'-TGGCACCCAGCACAATGAA-3' & / \\
\hline & Reverse & 5'-CTAAGTCATAGTCCGCCTAGAAGCA-3' & \\
\hline
\end{tabular}




\section{Immunohistochemistry staining}

The paraffin blocks specimens were cut in $4 \mu \mathrm{m}$ sections using a cryostat and collected on poly L-lysine-coated slides. TWEAK expression was analyzed with biotinylated antihuman TWEAK antibody (R\&D System Inc., USA) and phosphate-buffered saline (PBS) as blank control. The sections reacted with specific antibodies at $37^{\circ} \mathrm{C}$ for $2.5 \mathrm{~h}$. After washing with PBS for three times, the sections were incubated with biotinylated anti-goat immunoglobulins (\#SP-9003, Zhongshan Goldenbridge Biotechnology, Beijing, China) for $15 \mathrm{~min}$ at $37^{\circ} \mathrm{C}$ and washed with PBS to remove any unreacted antibodies. The sections were then treated with peroxidase-conjugated streptavidin for $15 \mathrm{~min}$, and washed with PBS again, then reacted with $\mathrm{DAB}$ in the presence of $3 \% \mathrm{H}_{2} \mathrm{O}_{2}$ to develop the color. The sections were counterstained with haematoxylin and mounted with glycerol.

\section{Western blotting}

Tissue $(n=12)$ was put in the homogenizer and the protein was extracted by RIPA Cell Lysis Buffer (Shanghai Biocolor BioScience \& Technology Company, China) following manufacturer's instructions. Four samples were from ectopic endometrium, 4 from eutopic endometrium and 4 from control endometrium. Samples containing $25 \mu \mathrm{g}$ of protein and a protein marker were subject to $15 \%$ sodium dodecyl sulfate-C polyacrylamide gel electrophoresis and were transferred to hydropholoic polyvinylidene fluoride transfer membrane (PVDF) membranes (Lot. 65421, Pall Corporation). These membranes were blocked for $2 \mathrm{~h}$ at room temperature in 10\% nonfat skim milk in PBS and Tween 20 (PBST). After blocking, the membrane was incubated at $4{ }^{\circ} \mathrm{C}$ overnight with biotinylated anti-human TWEAK antibody and mouse anti- $\alpha$-Tubulin (ZYMED Laboratories). The PVDF membranes were then washed again for three times. TWEAK was complexed with peroxidase-conjugated anti-goat IgG $(\mathrm{H}+\mathrm{L})$ and Tubulin antibody was complexed with peroxidase-conjugated anti-goat IgG $(\mathrm{H}+\mathrm{L})$ (Shanghai Yusen Biological Engineering Technology \& Services Corporation) for $1 \mathrm{~h}$ at room temperature. After that, the membranes were washed in PBST for four times, and the blot was visualized using the ECL detection system: BeyoECL Plus (P0018, Beyotime Institute of Biotechnology).

\section{Statistical analysis}

When needed, logarithmic transformation of the data was performed to homogenize variances and normalize the distribution. One-way ANOVA was used to detect differences of the relative transcript levels and protein levels of TWEAK between research group and control group. Statistical analysis was displayed as $\bar{x} \pm s$. Calculations were performed by SPSS software, version 11.0 (SPSS Inc., Chicago, IL). Significance was defined for $P<0.05$. 


\section{Results}

\section{Identification of TWEAK mRNA in human endometrium with RT-PCR}

TWEAK expression, as well as Fn-14 and GAPDH expression on the endometrial samples from eutopic, ectopic and control endometrium is shown in Figure 1. As we can see, TWEAK and Fn-14 were both detected on all the three kinds of endometrium, no matter it's from patients with endometriosis or without.

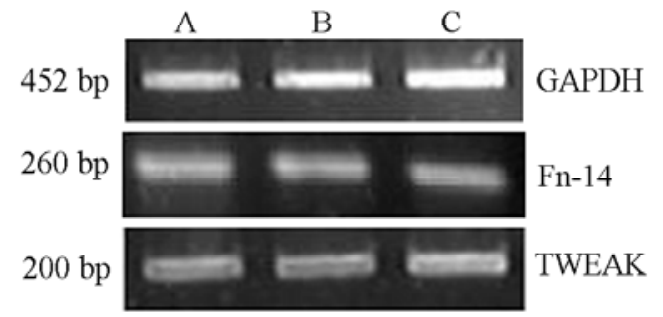

Figure 1 TWEAK and Fn-14 mRNA expression in human normal endometrium (A), EMs' ectopic endometrium (B) and eutopic site (C)

\section{Identification of TWEAK mRNA in human endometrium with Real-time PCR}

The relative TWEAK mRNA abundance of all the samples was analyzed by Real-time PCR and it is presented in Figure 2. The expression level of TWEAK was normalized by using $\beta$-actin. The data obtained showed that the average relative TWEAK mRNA abundance on ectopic site was much lower than that on eutopic endometrium $(P<0.05)$ and control endometrium $(P<0.05)$ in the secretory phase after normalization. Besides, TWEAK expressions on both eutopic endometrium and control endometrium in secretory phase were higher than those in proliferative phase. However, the Real-time PCR test did not suggest significant abnormalities of TWEAK expression between these two groups, no matter which phase they were.

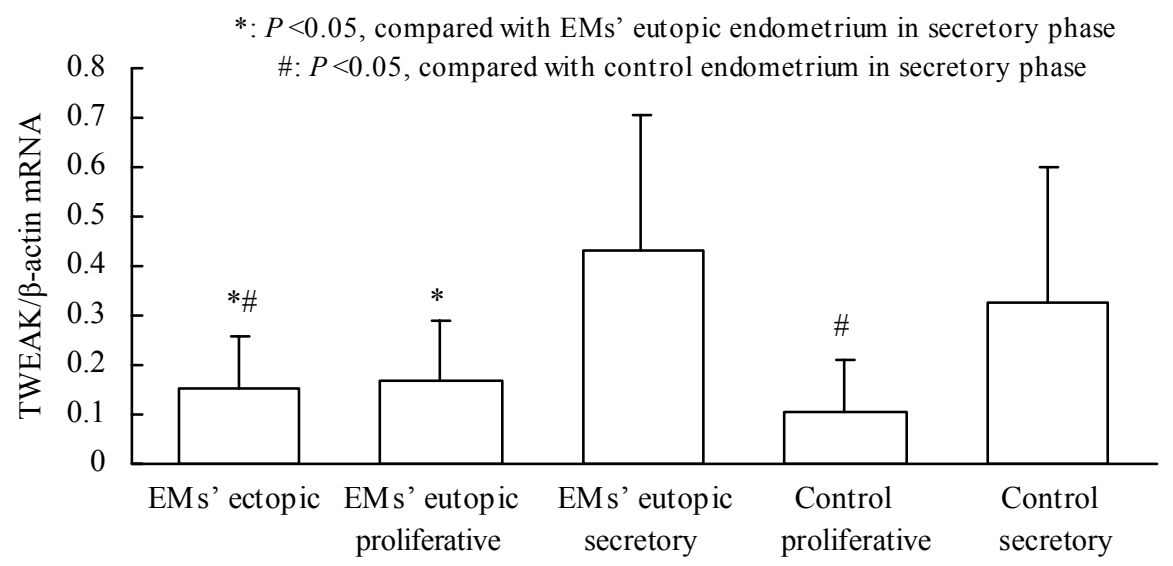

Figure 2 Comparison of TWEAK mRNA expression on EMs' eutopic, EMs' ectopic and control endometrium 


\section{Identification of TWEAK protein in human endometrium}

Figure 3 showed that TWEAK was indeed expressed on all the endometrium. It was produced both in the cytoplasm of glandular epithelium cells and stromal cells of endometrium. On control and eutopic endometrium, strong immunohistochemical staining could be detected in the glandular epithelium cells and stromal cells, whereas decreased staining was seen on ectopic endometrium. After normalization over tubulin, the value of TWEAK protein amounts on ectopic endometrium was much lower than that on eutopic site $(P<0.05)$
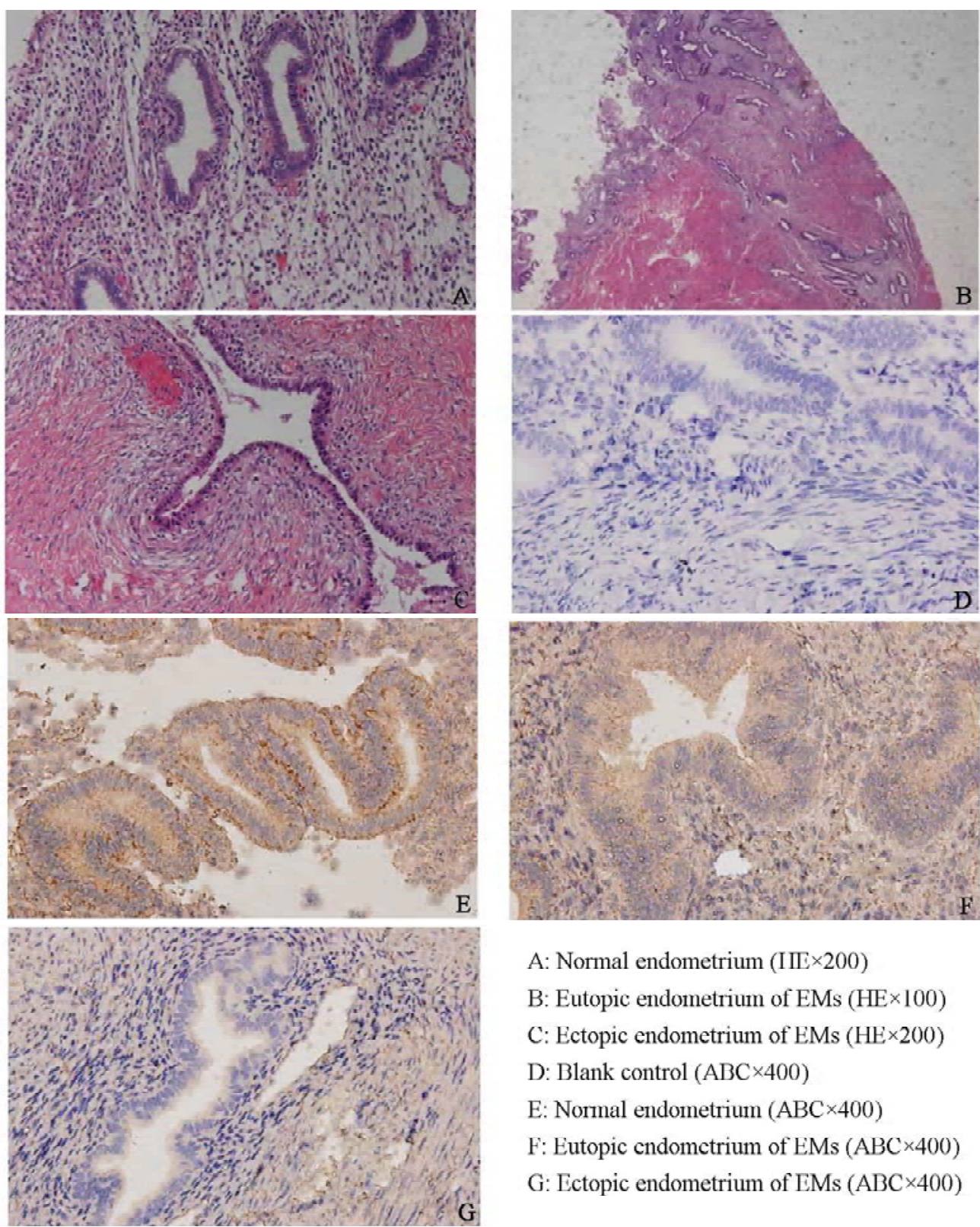
A: Normal endometrium ( $\mathrm{HE} \times 200)$
$\mathrm{B}$ : Eutopic endometrium of EMs $(\mathrm{HE} \times 100)$
C: Ectopic endometrium of EMs (HE $\times 200)$
D: Blank control $(\mathrm{ABC} \times 400)$
E: Normal endometrium $(\mathrm{ABC} \times 400)$
F: Eutopic endometrium of EMs $(\mathrm{ABC} \times 400)$
$\mathrm{G}$ : Ectopic endometrium of EMs $(\mathrm{ABC} \times 400)$

Figure 3 TWEAK protein localization in human normal, eutopic and ectopic endometrium 
and control endometrium (Figure 4). However, it seemed that there was no significant difference of TWEAK expression on eutopic endometrium when compared with control samples taken from patients without endometriosis.

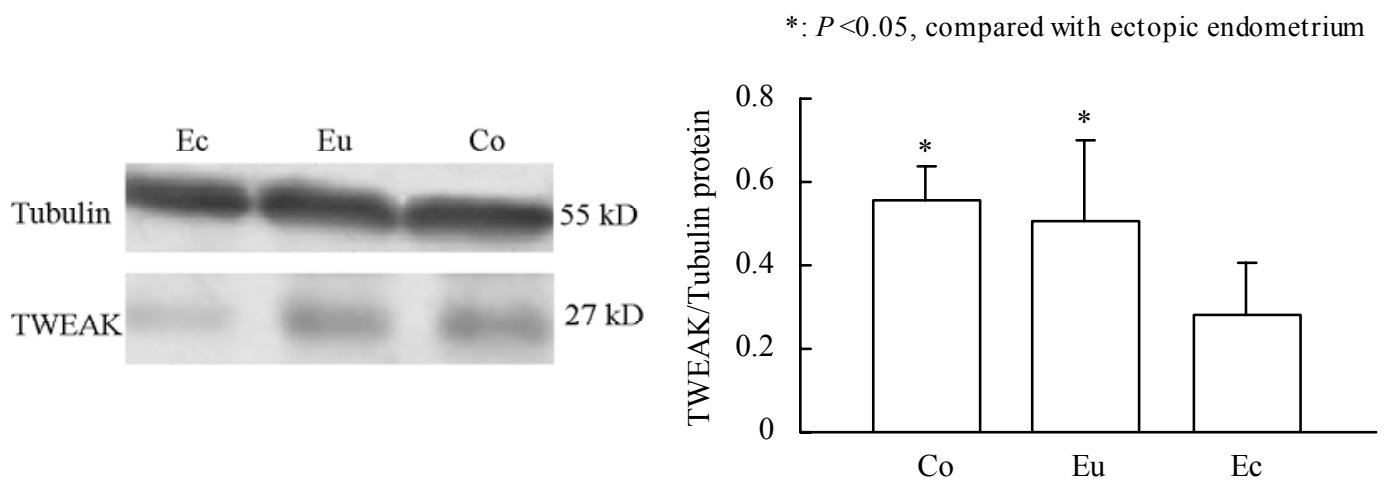

Figure 4 Expression of TWEAK protein on human normal (Co), eutopic $(\mathrm{Eu})$ and ectopic endometrium (Ec)

\section{Discussion}

Endometriosis is a common gynecologic disease, which most affects reproductive-aged women. About $20 \%-90 \%$ of women with endometriosis are associated with pelvic pain and infertility, and the frequency varies from $30 \%$ to $40 \%$ in women with infertility. The most widely accepted theory on the pathogenesis of endometriosis, proposed by Sampson, is that the disorder originates from retrograde menstruation of endometrial tissue sloughed through patent fallopian tubes into the peritoneal cavity ${ }^{[3]}$. After Sampson, varieties of theories, including metaplasia, altered cellular immunity, genetic basis, environmental basis and multifactorial mode of inheritance with interactions between specific genes and the environment have been brought up to explain the mechanism of endometriosis. Nevertheless, its etiology remains uncertain. Important advances have been made within the past few years that the abnormal behavior of immune system could be one of the leading roles in the process of this disease and a lack of adequate immune surveillance is considered as a cause of the disorder ${ }^{[4]}$.

Ligands of TNF family are expressed as type II membrane proteins ${ }^{[5]}$, and TWEAK is such a kind of ligand that comprises 249 amino acids and has a molecular weight of $27 \mathrm{kD}$. TWEAK is abundantly expressed in many places, such as human placenta, cytotrophoblast, placenta macrophage and placenta cell line ${ }^{[6]}$. With its diversity in sources, it has various sorts of receptors, through which it presents different biologic activities. Take Fn-14 for example, this cytokine has a high affinity with TWEAK, and interaction between TWEAK and Fn-14 could induce the expression of intercellular adhesion molecule 1(ICAM-1) on the cell surface and make human umbilical vein endothelial cells (HUVEC) secret cytokines, like interleukin-8 (IL-8) and monocyte chemotactic protein-1 (MCP-1) ${ }^{[7]}$. It has also been 
reported that they may transmit death signals and induce apoptosis through promoting the nuclear translocation of both classical and alternative NF- $\kappa B$ pathway subunits ${ }^{[8]}$. Nakayama et al. have observed apoptosis caused by the death signals from Fn14 receptors on all the cell surfaces when they were doing research on the TWEAK-sensitive tumor cell lines, whereas the cell death could be blocked completely by inhibiting the binding of TWEAK to Fn-14 with agonistic anti-death receptor $\mathrm{mAbs}^{[2]}$. Therefore, the apoptosis pathway induced by TWEAK may be mainly mediated by Fn-14. Meanwhile, the mouse and human versions of TWEAK are conserved with $93 \%$ amino acid identity in the receptor binding domain, which is usually seen on the FasL ${ }^{[5]}$. It indicates that TWEAK may fuction like FasL in the apoptosis. Our research has also observed lower expression of TWEAK on ectopic endometrium from patients with EMs, and we think that TWEAK involve in the apoptosis which may contribute to the development of EMs.

Compared with the samples taken from patients without EMs and eutopic endometrium from patients with EMs, both the levels of mRNA and protein of TWEAK were decreased on ectopic endometrium. These abnormalities may influence the apoptosis of endometrial cells through the mechanisms as follows. First of all, FasL is expressed on human endometrium throughout the menstrual cycle $\mathrm{e}^{[9-11]}$, and its proteins are primarily retained within the cell's Golgi apparatus and cytoplasmic vesicles during the late proliferative phase. When secretory phase comes, they are extruded as part of the cellular membranes, where Fas can bind FasL turning on apoptotic signals ${ }^{[11,12]}$. Therefore, under normal circumstances, FasL expression must be higher in secretory phase. Just as our research shows, TWEAK is expressed on the endometrium like FasL, and its concentration also rises in secretory phase. Down-regulation of TWEAK protein expression on ectopic endometrium suggests that it may facilitate ectopic endometrial tissue survive and allow implants to develop through less interaction with its death receptor, such as DR3 which is considered to influence the activation and apoptosis of the NF- $\kappa$ B pathway. With the impairment of the transmitting signals in the programmed cell death pathway, the percentage of apoptosis in endometrial cells is greatly reduced when they should have got into the program. And this implies that the number of surviving cells that enter the peritoneal cavity is larger and that escape from the surveillance of immune system is greater in women who develop EMs. Accumulated evidences suggest that apoptosis help to maintain cellular homeostasis by eliminating senescent cells from the functional layer of uterine endometrium during the late secretory and menstrual phase of the cycle ${ }^{[13]}$. What displays in our results shows down-regulation of TWEAK expression on ectopic site, suggesting that the survival ability of endometrial cells is stronger and these cells are capable of resisting the powerful immune system. But it still remains unknown that which the specific receptor is for TWEAK inducing apoptosis of endometrial cells. As far as we are concerned, TWEAK could induce cell death in $\mathrm{hFn}-14$ transfectants. Therefore, we suppose that TWEAK function in the development of EMs with Fn-14, which may be proved indirectly by our 
results that Fn-14 is expressed on all the three types of endometrium. Secondly, an increase of the $\mathrm{T}$ helper to $\mathrm{T}$ suppressor ratio on ectopic endometriotic issue and in peritoneal fluid is observed in women with EMs, whereas the T-lymphocyte numbers on eutopic endometrium seems to be unchanged ${ }^{[14,15]}$. The differences of immunological regulation between eutopic and ectopic endometrium indicate that ectopic endometrium should own a stronger antiapoptosis ability to implant outside. And associated with various sorts of cytokines and growth factors expressed abnormally in peritoneal fluid, the down-regulated expression of TWEAK on ectopic site must have been influenced by them, which could result in stronger capability against apoptosis. Moreover, it has been manifested that angiogenesis is essential for the development of EMs. IL-8, a chemokine that is a potent angiogenic factor, has been reported being elevated in the peritoneal fluid of women with EMs, and its level correlates with the severity of the disease ${ }^{[17,18]}$. Harada and his colleagues reported that the interaction between TWEAK and Fn-14 would induce HUVEC secret IL-8, suggesting that up-regulated expression of IL-8 in the peritoneal cavity could affect the expression of TWEAK on ectopic endometrium inversely, with whose down-regulation leading to the apoptosis level of endometrial cells on ectopic site decreasing further ${ }^{[7]}$. There are also experiments showing that TWEAK has a dual role in angiogenic regulation ${ }^{[19]}$. For example, when cooperating with basic fibroblast growth factor (bFGF), TWEAK induces the proliferation and migration of human endothelial cells and morphogenesis of capillary lumens. In contrast, without inhibiting VEGF-induced survival or proliferation, TWEAK antagonizes the morphogenic response of endothelial cells to vascular endothelial growth factor (VEGF). In conclusion, TWEAK has different functions when influenced by different factors and it involves in the development of EMs. However, we only detect Fn-14 expressions on the endometrium, but the relationship between TWEAK and Fn-14 still needs more in vitro experiments, so does that of TWEAK and IL-8.

Owing to the fact that TWEAK was defined just a few years ago, we have not got any report about the role of TWEAK in the development of EMs. Our data suggest that TWEAK, this small weak inducer of apoptosis, involves in the process of EMs. Its down-regulation on ectopic endometrium from patients with EMs could play a role in the initiation of this disease by causing reduced apoptosis and may contribute to the survival of the regurgitating endometrial cells into the peritoneal cavity and the development of EMs. Yet, more researches are needed for explanation of what its specific receptor is and which signal transduction pathway it takes part in when referring to the pathogenesis of EMs.

\section{References}

1. Kaplan MJ, Ray D, Mo RR, et al. TRAIL (Apo2 ligand) and TWEAK (Apo3 ligand) mediate CD4+ T cell killing of antigen-presenting macrophages. J Immunol, 2000, 164(6):2 897-904. 
2. Nakayama M, Ishidoh K, Kojima Y, et al. Fibroblast growth factor inducible 14 mediates multiple pathways of TWEAK-induced cell death. J Immunol, 2003, 170(1):341-8

3. Sampson JA. Peritoneal endometriosis due to the menstrual dissemination of endometrial tissue into the peritoneal cavity. Am J Obstet Gynecol, 1927, 14:422-69.

4. Dmowski WP. Immunological aspects of endometriosis. Int J Gynaecol Obstet, 1995, 50(Suppl 1):S3-10.

5. Chicheportiche Y, Bourdon PR, Xu HD, et al. TWEAK, a new secreted ligand in the tumor necrosis factor family that weakly induces apoptosis. J Biol Chem, 1997, 272(51):32 401-10.

6. Phillips TA, Ni J, Hunt JS. Death-inducing tumour necrosis factor (TNF) superfamily ligands and receptors are transcribed in human placentae, cytotrophoblasts, placental macrophages and placental cell lines. Placenta, 2001, 22(8-9):663-72.

7. Harada N, Nakayama M, Nakano H, et al. Proinflammatory effect of TWEAK/Fn-14 interaction on human umbilical vein endothelial cells. Biochem Biophys Res Commun, 2002, 299(3):488-93.

8. Saitoh T, Nakayama M, Nakano H, et al. TWEAK induces NF-kB2 p100 processing and long lasting NF$\kappa B$ activation. J Biol Chem, 2003, 278(38):36 005-12.

9. Garcia-Velasco J, Arici A, Zreick T, et al. Macrophage-derived growth factors regulate FasL expression in endometrial stromal cells: a role in endometriosis. Mol Hum Reprod, 1999, 5(7):642-50.

10. Yamashita H, Otsuki Y, Matsumoto K, et al. Fas ligand, Fas antigen and Bcl-2 expression in human endometrium during the menstrual cycle. Mol Hum Reprod, 1999, 5(4):358-64.

11. Song J, Rutherford T, Naftolin F, et al. Hormonal regulation of apoptosis and the Fas and Fas ligand system in human endometrial cells. Mol Hum Reprod, 2002, 8(5):447-55.

12. Otsuki Y. Apoptosis in human endometrium: apopototic detection methods and signaling. Med Electron Microsc, 2001, 34(3):166-73.

13. Dahmoun M, Boman K, Cajander S, et al. Apoptosis, proliferation, and sex hormone receptors in superficial parts of human endometrium at the end of secretory phase. J Clin Endocrinol Metab, 1999, 84(5):1 737-43.

14. Dmowski WP, Gebel HM, Braun DP. The role of cell-mediated immunity in pathogenesis of endometriosis. Acta Obstet Gynecol Scand Suppl, 1994, 159(Suppl):7-14.

15. Witz CA, Montoya IA, Dey TD, et al. Characterization of lymphocyte sub populations and T cell activation in endometriosis. Am J Reprod Immunol, 1994, 32(3):173-9.

16. Mettler L, Volkov NI, Kulakov VI, et al. Lymphocyte subsets in the endometrium of patients with endometriosis throughout the menstrual cycle. Am J Reprod Immunol, 1996, 36(6):342-8.

17. Arici A, Tazuke SI, Attar E, et al. Interleukin-8 concentration in peritoneal fluid of patients with endometriosis and modulation of interleukin-8 expression in human mesothelial cells. Mol Hum Reprod, 1996, 2(1):40-5.

18. Ryan IP, Tseng JF, Schriock ED, et al. Interleukin-8 concentrations are elevated in peritoneal fluid of women with endometriosis. Fertil Steril, 1995, 63(4):929-32.

19. Jakubowski A, Browning B, Lukashev M, et al. Dual role for TWEAK in angiogenic regulation. J Cell Sci, 2002, 115(Pt 2):267-74.

(Received on June 18, 2008) 